\title{
Studies on Bio-adhesion of Matrix Tablets: II. Comparison on Bio-adhesion Strength and Release Profiles of Theophylline Anhydrous and Its Sodium Glycinate Salt
}

\author{
Md. Saiful Islam¹, Md. Ziaur Rahman Khan ${ }^{2}$, Masuda Khatun ${ }^{1}$, J akir Ahmed \\ Chowdhury ${ }^{2}$ and Md. Selim Reza ${ }^{2}$ \\ ${ }^{1}$ Department of Pharmacy, University of Asia Pacific, Dhaka-1209, Bangladesh \\ ${ }^{2}$ Faculty of Pharmacy, University of Dhaka, Dhaka-1000, Bangladesh
}

\begin{abstract}
Controlled release matrix tablets of Theophylline anhydrous and Theophylline sodium glycinate were prepared with different types of bio-adhesive polymers e.g. HPMC 15 \& 50 cps, Gelatin, PVP K-30, Na-CMC and Xanthan gum. Tablets, prepared by direct compression method were subjected to in-vitro drug dissolution for 8 hours in $0.1 \mathrm{~N}$ hydrochloric acid ( $\mathrm{pH} 1.2)$ using a thermal shaker at $50 \mathrm{rpm}$ at $37 \pm 0.5^{\circ} \mathrm{C}$. The bio-adhesive property was investigated in terms of retention time following in-vitro wash-off method by Lehr et al. The anhydrous and corresponding salt form was used to investigate whether physical variation of Theophylline molecule can cause any change in release and bio-adhesion property or not. In most of the cases it is found that salt form releases greater percentage of active drug than its corresponding anhydrous form. Irrespective of drug's physical property and amount of polymer, bio-adhesive strength of Xanthan gum was proved to be maximum followed by HPMC- 50 cps, Na-CMC and HPMC- 15 cps, whereas gelatin and PVP K-30 showed little or poor muco-adhesion. Xanthan gum, Na-CMC and HPMC based formulations showed nearly zero-order release; on the contrary PVP K-30 based formulation showed a burst release within one hour of dissolution.
\end{abstract}

Key words: Bio-adhesion, Theophylline anhydrous, Theophylline sodium glycinate, Retention time, Release profile.

\section{INTRODUCTION}

Orally administered controlled release dosage form shows mainly two adversities; too short gastric retention time (GRT) and unpredictable gastric emptying time. A relatively brief GI transit time of most of the products (8-12 hours) impedes the formulation of single daily dosage forms. These problems can be overwhelmed by altering the gastric emptying, which is affected by age, sex, and health condition of a subject. It is therefore desirable to formulate a controlled release dosage form that gives an extended GI residence time. Various approaches have been worked out to improve the retention of an

Correspondence to: Md. Selim Reza

Tel: 880-2-8612069; Fax: 880-2-8615583

E-mail: selimreza_04@yahoo.com

Dhaka Univ. J. Pharm. Sci. 10(1): 1-7, 2011 (June) oral dosage form in the stomach including bioadhesive systems. ${ }^{1}$ Bioadhesive systems are usually exploited to localize a delivery device within the cavity of body to improve the drug absorption process in site-specific manner. ${ }^{2}$ Mucous is a network of mucin glycoproteins. At physiological $\mathrm{pH}$ the mucin network has a net negative charge due to the presence of sialic acid having pKa 2.6 and presence of sulphate residues. ${ }^{3}$ Bioadhesive polymers are usually macromolecules within numerous hydrophilic functional groups (e.g. carboxyl, hydroxyl, amide, and sulphate groups) that can form hydrogen bond with sialic acid. These polymers also form viscous layers when hydrated with water that increases the retention time on mucosal surfaces and may lead to adhesive interaction. ${ }^{4}$ In our previous study 
Theophylline anhydrous was used as a model drug to develop, characterize and evaluate the bio-adhesive matrix tablets employing various bio-adhesive polymers for prolonged gastrointestinal absorption. ${ }^{5}$ Our present study is designed to actually study the capacity of hydrophilic polymers as release retarding ingredient and muco-adhesive agent over drug molecule in its anhydrous and salt form in terms of dissolution study, retention time and subsequent data analysis.

\section{MATERIALS AND METHODS}

Pharmaceutical grade Theophylline anhydrous and Theophylline sodium glycinate were gifts from Square Pharmaceuticals Limited. Hydroxypropylmethylcellulose (HPMC 15 cps and $50 \mathrm{cps}$ ), Gelatin, PVP K-30, Sodium carboxy methylcellulose (Na$\mathrm{CMC}$ ) and Xanthan gum were gift samples from Loba Chemie Pvt. Ltd, India. Magnesium stearate and Aerosil were obtained from Hanau Chemical Ltd. (Japan). For determining bio-adhesive strength, gastric mucosa of cow was obtained from a local slaughterhouse in Dhaka. All other reagents employed were of analytical or pharmaceutical grade.

Preparation of matrix tablets. Direct compression technique was employed for the preparation of Theophylline loaded matrix tablets. Table 1 represents the formulation of matrix tablets with their code. Amount of active ingredient is constant $(100 \mathrm{mg})$ in all cases. Total weight of tablet was $406 \mathrm{mg}$ (F-1a to F-1f \& F-3a to F-3f) and 609 mg (F-2a to F-2f \& F-4a to F-4f). The required amount of active and polymers were weighted separately and mixed thoroughly in a drum blender. Matrix tablets were prepared by Perkin-Elmer hydraulic press applying a compression force of 5 ton. Before compression surface of die and punch was lubricated with magnesium stearate. All the tablets were then stored in air tight containers at room temperature for further investigation.

In vitro dissolution studies. In vitro drug release studies of the prepared matrix tablets were carried out in a thermal shaker (Memmert) apparatus equilibrated at $37+/-0.5^{\circ} \mathrm{C}$ at $50 \mathrm{rpm}$. The dissolution study was performed for 8 hours in $250 \mathrm{ml}$ of $0.1 \mathrm{~N}$ hydrochloric acid $(\mathrm{pH}$ 1.2) in triplicate. The dissolution samples were collected at every 30 minutes interval and replaced with an equal volume of dissolution fluid $(10 \mathrm{ml})$. Sample solution was analyzed for Theophylline at $271 \mathrm{~nm}$ as mentioned in USP and BP by an UV spectrophotometer (Shimadzu, Japan). The amount of drug present in the samples was calculated from the equation of standard curve. Drug dissolved at specified time period was plotted as percent release versus time (hours) curve.

Bio-adhesive testing by in-vitro wash-off test. The bio-adhesive property of the prepared tablets was evaluated by an in-vitro adhesion testing method known as wash-off method described by Lehr et al. (1990). ${ }^{6}$ Freshly excised pieces of intestinal mucosa (4X3 inch) from cow were mounted on the stainless steel slide, connected with a suitable support. Three tablets were placed onto wet rinsed tissue specimen and immediately thereafter the support was hung to the arm of the USP tablet disintegrating test machine. When the disintegrating machine was operated, the tissue specimen was given a slow, regular up-anddown movement in the gastric fluid $(\mathrm{pH} 1.2)$ at $37^{\circ} \mathrm{C}$ contained in a one liter vessel of the machine.

Data treatment. The dissolution data was also fitted according to well-known exponential Eq. (1), which is often used to describe the drug release behavior from polymeric systems.

$$
\mathrm{M}_{\mathrm{t}} / \mathrm{M}_{\propto}=\mathrm{Kt}^{\mathrm{n}}
$$

Where $M_{t} / M_{\infty}$ is the fractional (0.1-0.7) drug release at time $\mathrm{t} ; \mathrm{K}$ is a constant incorporating the properties of macromolecular polymeric systems and the drug while $\mathrm{n}$ is a kinetic constant, which depends on and is used to characterize the transport mechanism. The value of $\mathrm{n}$ for a tablet, $\mathrm{n}=0$ to 0.45 for Fickian (Case 1) release, $>0.45$ but $<0.89$ for non-Fickian (Anomalous) release and 0.89 for case II (zero-order) release and $>0.89$ for super case II type of release. ${ }^{7}$ Case II transport generally refers to dissolution of the polymeric matrix due to the relaxation of the polymeric chain and anomalous transport refers to the summation of both diffusion and dissolution controlled drug release. From the 
above equation $\mathrm{n}$ value for different formulation was calculated to identify the drug release mechanism. Although the constant K used in Eq (1) is one of the measures of drug release rate, it should not be used for comparison because there is different kinetics in different experimental condition. Therefore to characterize drug release rate in different experimental condition, Mean Dissolution Time (MDT) was calculated from dissolution data according to Mockel and Lippold (1993) Eq. (II). ${ }^{8}$

$$
\mathrm{MDT}==\mathrm{n} /(\mathrm{n}+1) \mathrm{k}^{-1 / \mathrm{n}}
$$

The value of release exponent (n) and Mean Dissolution Time (MDT) calculated according to Eq. (II) is depicted in table 2.

\section{RESULTS AND DISCUSSION}

The serum half-life of Theophylline in a healthy, nonsmoking asthmatic adult is 6 to 12 hours. ${ }^{9}$ Theophylline has been shown to be well adsorbed through the length of gastrointestinal tract and produce peak plasma drug concentration within 2 hours. The use of rapid release Theophylline has been declined due to higher incidence of side effects resulting from rapid absorption. Theophylline has a short elimination half-life, which also encourages the drug to be formulated in sustained release dosage form. Due to its rapid elimination and narrow therapeutic index, this drug is indeed a suitable candidate to be formulated into sustained release dosage form. ${ }^{10}$

Effect of HPMC-15cps and HPMC-50cps on the release and bio-adhesive strength of theophylline anhydrous and its glycinate salt. Figure 1 (A) and Figure 1 (B) show the effect of different concentration of HPMC-15cps and HPMC50 cps on drug release characteristics from both Theophylline anhydrous and its salt form. Differences in release pattern was observed between the formulations of F-1a, F-2a, F-3a \& F-4a and F-1b, F-2b, F-3b \& F-4b. Theophylline anhydrous loaded F-1a and F-2a contain 300 and $500 \mathrm{mg}$ of HPMC-15cps and released 55.08 and $37.25 \%$ of active after 8 hours of dissolution period. Whereas Theophylline sodium glycinate loaded matrix tablets
(F-3a and F-4a) with same polymer amount released 63.89 and $43.03 \%$ of active after same time period. Again Theophylline anhydrous loaded F-1b and F-2b contain 300 and $500 \mathrm{mg}$ of HPMC-50 cps and released 51.86 and $40.57 \%$ of active molecule after 8 hours of dissolution period, while theophylline sodium glycinate loaded matrix tablets (F-3b and F4b) with same polymer amount released 52.19 and $59.70 \%$ of active after same time period. No formulation exerted any initial burst release. The ability of HPMC-15 cps to prevent burst release was also claimed by Reza et al. ${ }^{11}$ It is clearly evident that drug release was decreased with the increase in polymer loading. In both cases salt forms experienced higher dissolution due to its higher solubility in aqueous environment. HPMC- 15 cps is a class of hydrophilic matrix system which when comes in contact with liquid dissolution medium, forms fast and strong viscous gel to control initial drug release. ${ }^{11}$ While HPMC could potentially retard the release of a soluble drug, it could also facilitate the release of relatively insoluble drug due to its solubilizing effect. ${ }^{11}$ The hydrated gel matrix formed by HPMC is involved in the phenomenon of bioadhesion. Bio-adhesive strength was determined in triplicate using in-vitro wash-off method. F-1b, F-2b, F-3b and F-4b retained on the mucosa for 5.79, 5.69, 5.96 and 5.9 hours respectively. In contrast F-1a, F2a, F-3a and F-4a hold on to the mucosa for 0.36, $0.54,0.48$ and 0.43 hours respectively. Lower HPMC grade exerted short retention period with no significant difference between anhydrous and its salt form. Increment in polymer amount did not bring any significant and correlated data. Higher HPMC grade exerted a very high retention time compared to HPMC-15 cps possibly due to its more concentrated gel layer.

Effect of gelatin on the release and bioadhesive strength of theophylline anhydrous and its glycinate salt. Figure 1 (C) elaborates the effect of different concentration of gelatin on drug release characteristics from both Theophylline anhydrous and its salt form. TSG matrix tablets (F-3c and F-4c) with 300 and $500 \mathrm{mg}$ of gelatin released 74.32 and $94.43 \%$ of active after 8 hours of dissolution. But in 
case of TA loaded matrix tablet F-1c containing 300 mg of gelatin released $84.45 \%$ of drug after 8 hours of dissolution whereas F-1c with higher polymer content exhibited a burst release within 2 hours. A contradictory result compared to HPMC has been obtained that higher polymer content caused increased release for both anhydrous and salt form.
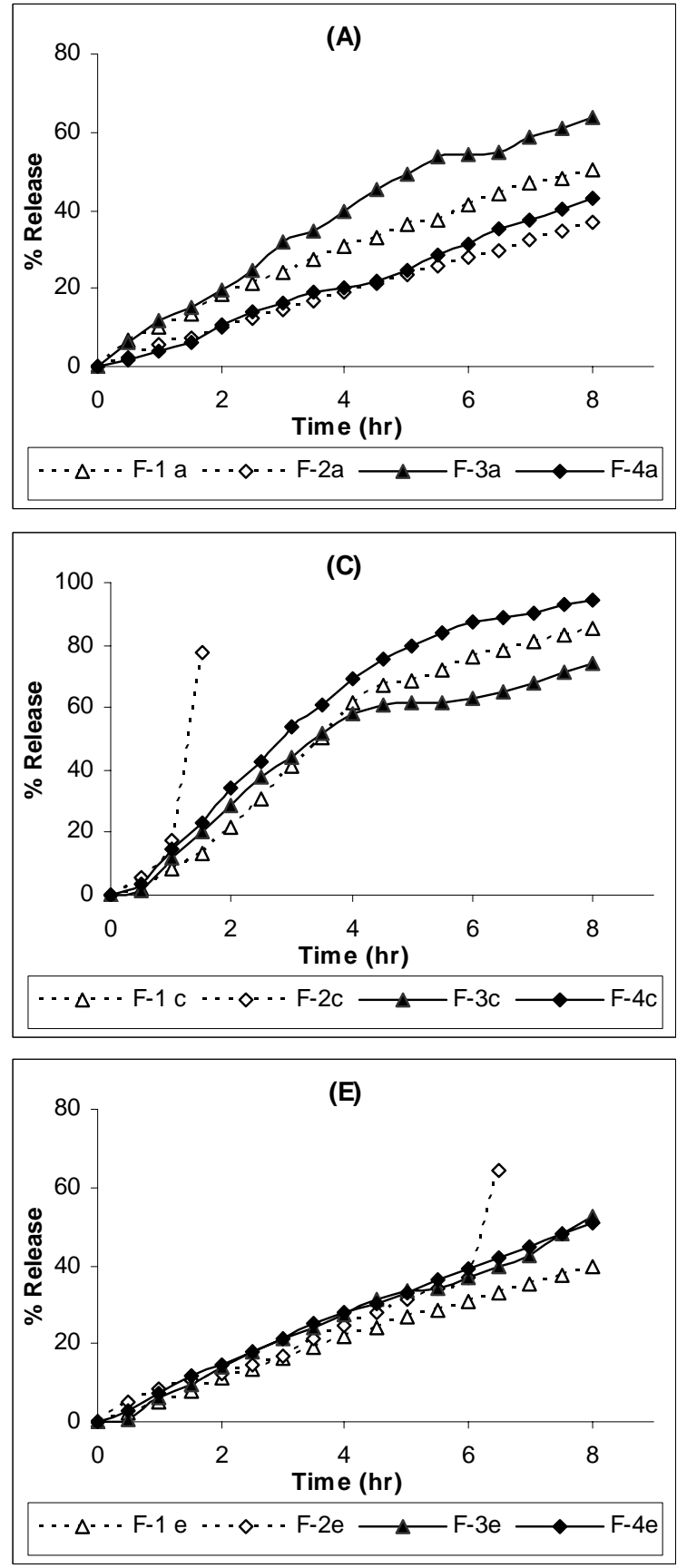

Although in case of $300 \mathrm{mg}$ polymer loading more soluble salt form released slightly higher amount of drug than its anhydrous form up to initial $4 \mathrm{hr}$. However, gelatin is categorized as poor bio-adhesive polymer at both $300 \mathrm{mg}$ and $500 \mathrm{mg}$ polymer loading as tablets were swapped within very first minute. ${ }^{12}$
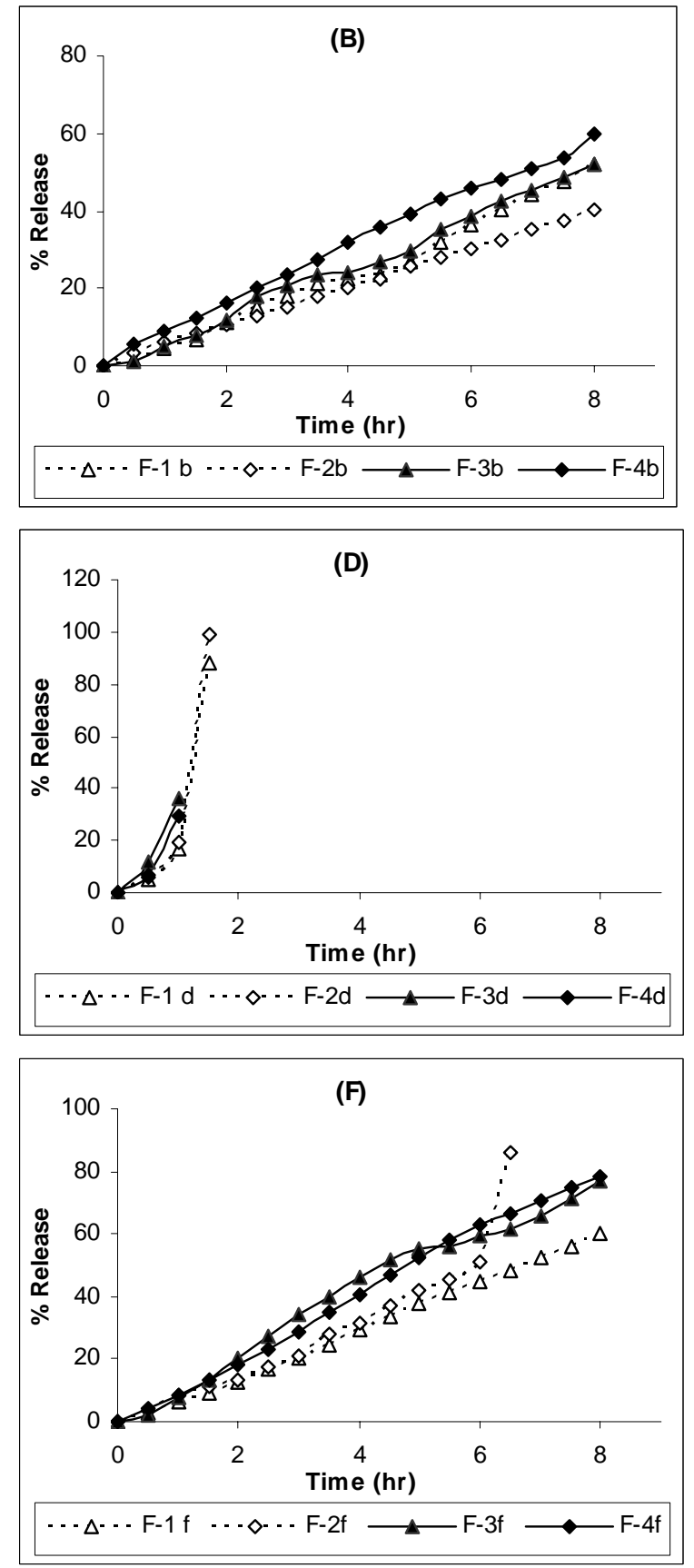

Figure 1. Zero-order release profile of matrix tablets using Theophylline anhydrous (--------) and glycinate ( — ) (A) HPMC-15 cps (B) HPMC-50 cps (C) Gelatin (D) PVP K-30 (E) Na-CMC (F) Xanthan gum 
Table 1. Formulations (mg) of Theophylline anhydrous (TA) or Theophylline sodium glycinate (TSG) loaded matrix tablets.

\begin{tabular}{lcccccccccccc}
\hline & F1a/ & F1b/ & F-1c/ & F-1d/ & F-1e/ & F-1f/ & F-2a/ & F-2b/ & F-2c/ & F-2d/ & F-2e/ & F-2f/ \\
& F-3a & F-3b & F3c & F-3d & F-3e & F-3f & F-4a & F-4b & F-4c & F-4d & F-4e & F-4f \\
TA / TSG & 100 & 100 & 100 & 100 & 100 & 100 & 100 & 100 & 100 & 100 & 100 & 100 \\
HPMC 15 cps & 300 & - & - & - & - & - & 500 & - & - & - & - & - \\
HPMC 50cps & - & 300 & - & - & - & - & - & 500 & - & - & - & - \\
Gelatin & - & - & 300 & - & - & - & - & - & 500 & - & - & - \\
PVP K-30 & - & - & - & 300 & - & - & - & - & - & 500 & - & - \\
Na-CMC & - & - & - & - & 300 & - & - & - & - & - & 500 & - \\
Xanthan gum & - & - & - & - & - & 300 & - & - & - & - & - & 500 \\
Aerosil & 4 & 4 & 4 & 4 & 4 & 4 & 4 & 4 & 4 & 4 & 4 & 4 \\
Mg-Stearate & 2 & 2 & 2 & 2 & 2 & 2 & 2 & 2 & 2 & 2 & 2 & 2 \\
Total & 406 & 406 & 406 & 406 & 406 & 406 & 506 & 506 & 506 & 506 & 506 & 506 \\
\hline
\end{tabular}

Table 2. Zero order drug release rate (K in \% release/time), kinetic constant (n), Mean Dissolution Time (MDT), Time to release $50 \%$ of drug $\left(T_{50 \%}\right)$ from different Theophylline loaded matrix tablets.

\begin{tabular}{cccccccccc}
\hline Formulation & $\mathrm{K}$ & $\mathrm{n}$ & $\begin{array}{c}\mathrm{MDT} \\
(\mathrm{hr})\end{array}$ & $\begin{array}{c}\mathrm{T}_{50 \%} \\
(\mathrm{hr})\end{array}$ & Formulation & $\mathrm{K}$ & $\mathrm{n}$ & $\begin{array}{c}\text { MDT } \\
(\mathrm{hr})\end{array}$ & $\begin{array}{c}\mathrm{T}_{50 \%} \\
(\mathrm{hr})\end{array}$ \\
\hline F-1a & 6.07 & 0.773 & 8.20 & 7.67 & F-3a & 8.07 & 0.863 & 6.45 & 6.23 \\
F-1b & 6.43 & 1.131 & 7.79 & 7.96 & F-3b & 6.59 & 1.085 & 7.49 & 7.60 \\
F-1c & 11.90 & 1.142 & 4.02 & 4.11 & F-3c & 9.28 & 0.822 & 1.59 & 1.61 \\
F-1e & 6.00 & 0.965 & 9.91 & 9.83 & F-3e & 6.34 & 0.967 & 7.82 & 7.77 \\
F-1f & 7.68 & 1.114 & 6.53 & 6.65 & F-3f & 9.78 & 1.057 & 4.83 & 4.88 \\
F-2a & 4.59 & 0.932 & 11.41 & 11.24 & F-4a & 5.42 & 1.095 & 8.92 & 9.06 \\
F-2b & 4.95 & 0.925 & 11.17 & 11.13 & F-4b & 7.21 & 0.923 & 6.79 & 6.68 \\
F-2c & 48.81 & 0.884 & 1.76 & 1.85 & F-4c & 12.58 & 0.884 & 3.30 & 3.22 \\
F-2e & 7.35 & 0.840 & 11.23 & 10.83 & F-4e & 6.08 & 0.910 & 7.96 & 7.79 \\
F-2f & 10.36 & 1.031 & 7.18 & 7.25 & F-4f & 10.29 & 1.081 & 4.90 & 4.97 \\
\hline
\end{tabular}

Effect of PVP K-30 on the release and bioadhesive strength of theophylline anhydrous and its glycinate salt. The release profile of theophylline from both of TA and TSG from PVP K-30 based matrix tablets is depicted in figure 1 (D). This polymer exerted initial burst release within hour irrespective of polymer amount or physical nature of drug. Povidone, being highly soluble in aqueous environment, is leached slowly from the matrix generating numerous pores and channels through which the drug is released by diffusion process. ${ }^{10}$ Moreover, theophylline is a highly water-soluble drug with pKa value 8.6. ${ }^{13}$ Both effects contribute to the initial burst release. Retention capacity was also insignificant, having a retention time of not more than few seconds.

Effect of Na-CMC on the release and bioadhesive strength of theophylline anhydrous and its glycinate salt. The release profile of theophylline from both of TA and TSG from Na-CMC based matrix tablets is depicted in figure 1 (E). TA loaded F-1e and F-2e contains 300 and $500 \mathrm{mg}$ of Na-CMC and released $39.54 \%$ after 8 hrs and $64.17 \%$ after 6.5 hrs of dissolution period. Whereas TSG loaded matrix tablets (F-3e and F-4e) with same polymer amount released 52.47 and $51.02 \%$ of active after same time period. In general the release pattern was almost zero-order throughout the dissolution period except F-2e as it lost the control over drug release after 6 hrs. These tablets showed significant bioadhesive strength specially the anhydrous form in $300 \mathrm{mg}$ polymer loading which exerted a retention time of more than 5.5 hour. Other formulations like F-2e, F-3e and F-4e retained for 2.64, 3.69 and 3.72 hours. The use of Na-CMC in bio-adhesive drug delivery system has been reported earlier. ${ }^{14}$

Effect of xanthan gum on the release and bioadhesive strength of theophylline anhydrous and 
its glycinate salt. The release profile of theophylline from both of TA and TSG from xanthan gum based matrix tablets is depicted in figure 1 (F). TA loaded F-1f and F-2f contains 300 and $500 \mathrm{mg}$ of xanthan gum and released 59.81 and $60.42 \%$ of active after 8 hours of dissolution period. Whereas TSG loaded matrix tablets (F-3f and F-4f) with same polymer amount released 76.94 and $78.23 \%$ of active after same time period. In general the release pattern was almost zero-order throughout the dissolution period except F-2f as it lost the control over drug release after 6 hrs. These tablets showed significant bioadhesive strength for more than $8 \mathrm{hrs}$ and above. Retention time of $8.13,10.5,6.9$ and 9.83 were obtained for F-1f, F-2f, F-3f and F-4f respectively. It was found that higher polymer content exhibited higher retention time whereas TSG loaded tablets showed higher retention than corresponding anhydrous loaded tablets. Among all formulations irrespective of polymer amount and drug's chemical entity xanthan gum exhibited highest retention time followed by HPMC-50 cps, Na-CMC and HPMC-15 cps. On contrary gelatin and PVP K-30 proved to be ineffective muco-adhesive agents.

Release kinetics. To know the mechanism of drug release from formulations, the data were treated according to zero-order (cumulative amount of drug release vs time), first order (log cumulative percentage of drug remaining vs time), Higuchi's (cumulative percentage of drug released vs SQRT of time) model and korsemeyer et al's (log cumulative percentage of drug released vs log time) equation. Due to insufficient data points PVP K-30 based formulations were not incorporated in this calculation. When the data were plotted according to zero-order equation all formulations except gelatin based tablets $\left(R^{2}=0.79-0.95\right)$ showed a strong linearity $\left(\mathrm{R}^{2}>0.99\right)$ (Table 2$)$. In case of Higuchi's model a fair linearity was obtained $\left(\mathrm{R}^{2}>0.94-0.99\right)$.

To confirm the drug release mechanism, the data were fit into Korsemeyer's equation. All the formulations showed strong linearity $\left(\mathrm{R}^{2}>0.97\right)$ with a comparatively high slope (n) value within the range of 0.773- 1.131 except gelatin based tablets. These results are indicative of case II (zero-order) and super case II type of release. ${ }^{7}$ The slope of the regression line from zero-order plot indicates drug release rate.

From the slope value of the Korsemeyer equation, MDT was calculated which is presented in table 2 along with $\mathrm{T}_{50 \%}$ and zero-order Drug release rate. MDT value is used to characterize the drug release rate from dosage form and the retarding efficacy of the polymer. A higher value of MDT indicates a higher drug retarding ability and viceversa. For different formulation maximum MDT values were obtained from Na-CMC and HPMC (15 cps \& 50 cps) followed by xanthan gum and gelatin. Conversely highest drug release rate was obtained from gelatin based formulations.

According to USP specification, for controlled drug delivery system (tablet/capsule), at time interval to $0.25 \mathrm{D}, 20-50 \%$ drug will be dissolved, at time equal to $0.5 \mathrm{D}, 45-75 \%$ dissolved and thereafter at any time up to $1.0 \mathrm{D}$, not less than $75 \%$ of drug will be dissolved where $\mathrm{D}$ is the labeled usual dosing frequency or interval. ${ }^{15}$ With this objective in view xanthan gum based tablet shows drug release according to USP specification.

\section{CONCLUSION}

Bio-adhesive studies and subsequent release profile were performed on matrix tablets containing either Theophylline anhydrous or Theophylline sodium glycinate to evaluate the performance of active when remain in two different physical forms. From pharmaceutical and biopharmaceutical viewpoint the most potential polymer should be one that possesses strong bio-adhesive property (drug is dissolved before dosage form leaves mucosa), sufficient release retarding capacity and provides a release profile that meets USP specification. Xanthan gum, HPMC and Na-CMC have been proved eligible to be used in bio-adhesive sustained release dosage form with above properties. Whereas gelatin possesses only sustaining property with little bioadhesive characteristics and PVP K-30 lacks both properties. Judicial selection of the mentioned hydrophilic polymer may lead to an optimum 
formulation with desirable distinctiveness. However, further studies in this context should be carried out to establish stability and reproducibility of this dosage form. Scopes like using X-ray and Gama scintigraphy should be explored to find the real bioadhesion in animals. In vitro- in vivo correlation should also be performed to assess the efficacy of this mucoadhesive dosage form.

\section{REFERENCES}

1. Vyas, S.P., and Khar, R.K. 2002. Controlled Drug Delivery $1^{\text {st }}$ Edn. Jain, M.K., Delhi, p. 199.

2. Wilson, C.G., Washington, N. 1989. Biological barriers to drug absorption. In: Physiological Pharmaceutics. Ellis Harwood, Chickestar, p. 47.

3. Schachter H. and Williams D. 1982.Biosynthesis of mucous glycoproteins. Adv. Exp. Med. Biol. 144. 3.

4. Hannah, B., 2004. Novel bio-adhesive formulations in drug delivery. Presented at 2004 British pharmaceutical conference on drug delivery to upper GI tract, particularly to esophagus. Medicines research unit, Aston University, Birmingham, B47ET, UK.

5. Shamsuddin, A.P., Khan, Z.R., Chowdhury, J.A., Reza, S. 2006. Studies on bio-adhesion of matrix tablets: I. Release profile of theophylline anhydrous. Dhaka Univ. J. Pharm. Sci. 5, 33-37.

6. Lehr, C.M., Bowstra, J.A., Tukker, J.J. and Junginer, H.E. 1990. Intestinal transit of bioadhesive microspheres in an insitu loop in the rat. J. Contr. Rel. 13, 51-62.
7. Ritger, P.L., and Peppas, N.A. 1987. A simple equation for description of solute release II. Fickian and anomalous release for swellable devices. J. Contr. Rel. 5, 37-42.

8. Mockel, J.E., and Lippold, B.C. Zero-order release from hydrocolloid matrices. Pharm. Res. 90, 1066-1070.

9. Theophylline information from Drugs Update. 2011. www.drugsupdate.com/generic/view/519.

10. Reza, S., Quadir, M.A., Haider, S.S. 2002. Development of theophylline sustained release dosage form based on kollidon SR. Pak. J. Pharm. Sci. 115, 63-70.

11. Lee, B.J., Ryu, S.G., and Cui, J.H. 1999. Formulation and release characteristics of hydroxypropylmethylcellulose matrix tablet containing melatonin. Drug Dev. Ind. Pharm. 25, 493-501.

12. Charrueau, C., Arnaud, P., Durieux, J., Allian, P., and Chaumeil, J.C. 1993. Oropharyngyal decontamination: bioadhesive powder mixture formulation. I. Choice of the bioadhesive material. J. Contr. Rel. 26, 49-57.

13. The Pharmaceutical Codex, (Lund, W., Ed), the Bath Press, Bath, Avon, p. 1068.

14. Rhodes, C.T., Jimenez-castellanos M.R. and Zia, H. 1994. Design and testing in vitro of a bioadhesive and floating drug delivery system for oral application. Int J. Pharm. 105, 65-70

15. Banker, U. 1992. Dissolution of modified release dosage forms. In: Pharmaceutical dissolution testing, $1^{\text {st }}$ Edn. Marcel-Dekker Inc, New York, p. 322. 